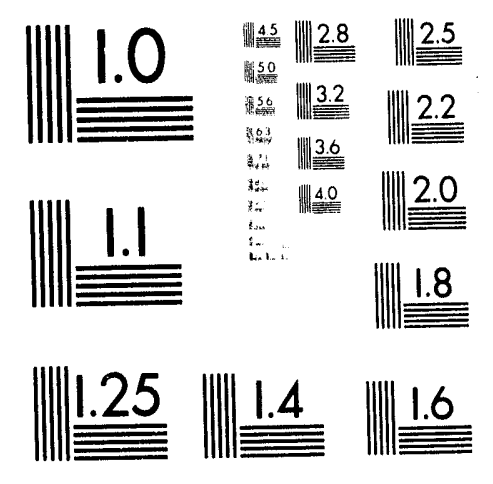



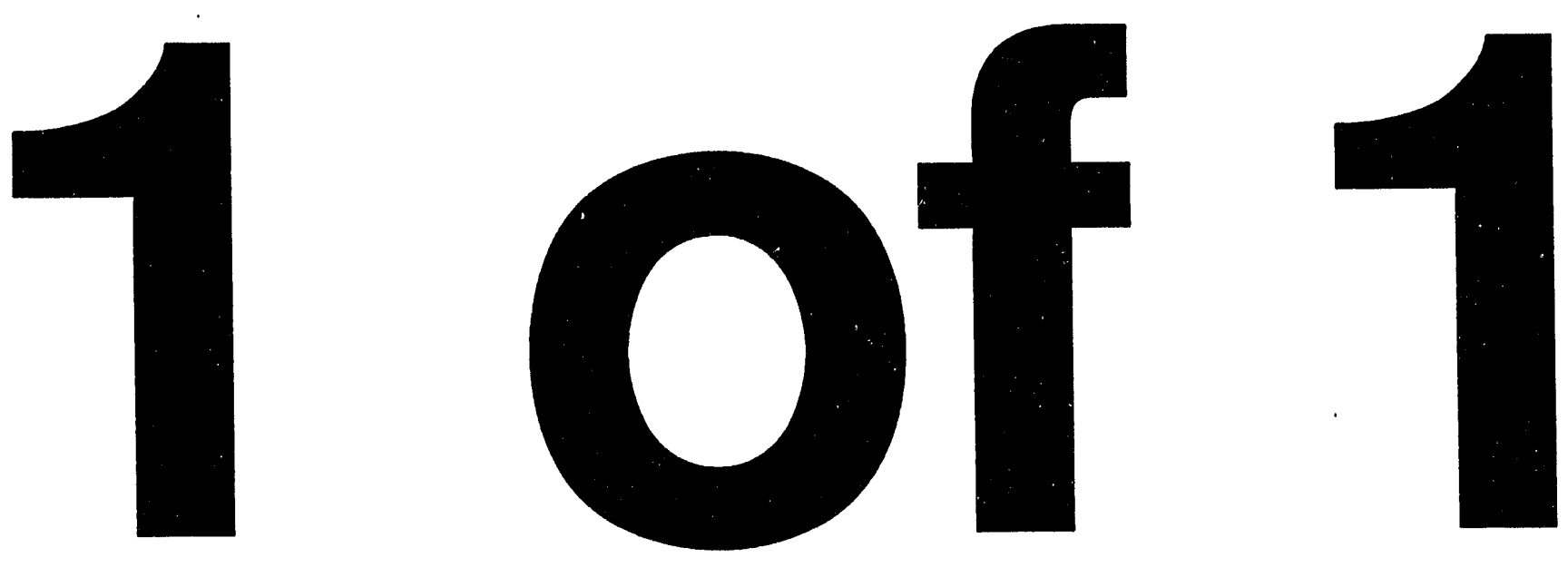


\title{
IMPROVED MEASUREMENT ACCURACY IN A LONG TRACE PROFILER: COMPENSATION FOR LASER POINTING INSTABILITY
}

\author{
S. Irick
}

\author{
Advanced Light Source \\ Accelerator and Fusion Research Division \\ Lawrence Berkeley Laboratory \\ University of California \\ Berkeley,CA 94720
}

August 2, 1993

Paper Presented at the 8th National Conference on Synchrotron Radiation Instrumentation, Gaithersburg, Maryland, USA, 8/23/93-8/26/93. 


\title{
Improved Measurement Accuracy in a Long Trace Profiler: Compensation for Laser Pointing Instability
}

\author{
Steven C. Irick \\ Lawrence Berkeley Laboratory \\ University of California \\ 1 Cyclotron Road \\ Berkeley, Ca 94720
}

\begin{abstract}
Laser pointing instability adds to the error of slope measurements taken with the Long Trace Profiler ${ }^{1}$ (LTP). As with carriage pitch error, this laser pointing error must be accounted for and subtracted from the surface under test (SUT) slope measurement. In the past, a separate reference beam (REF) allowed characterization of the component of slope error from carriage pitch. However, the component of slope error from laser pointing manifests itself differently in the SUT measured slope.

An analysis of angle error propagation is given, and the effect of these errors on measured slope is determined. Then a method is proposed for identifying these errors and subtracting them from the measured SUT slope function. Separate measurements of carriage pitch and laser pointing instability isolate these effects, so that the effectiveness of the error identification algorithm may be demonstrated.
\end{abstract}

\section{Introduction}

Ever since the LTP was first used for measuring long mirrors of arbitrary figure, there has been an on-going effort to increase the accuracy and reduce inherent noise of this instrument. The first LTP built by Takacs et al. ${ }^{2}$ provided a means of measuring this class of optical components that no commercially available instrument could provide. At that time it was impressive to have slope features in the sub-milliradian (mrad) regime revealed. 
More recently, high-brightness synchrotrons have pushed requirements of optical components in beamlines to preserve the brightness. Third-generation synchrotron beamlines have produced designs which specify slope errors of less than one microradian ( $\mu \mathrm{rad})$ rms. In order to achieve this goal, a straightness reference $^{3}$ was employed, which compensated for errors in carriage movement. An improved air bearing stage, environment control, and improved mounting methods ${ }^{4}$ also reduced the noise level, so that slope errors of $160 \mathrm{~mm}$ long mirrors could be measured with accuracies better than $1 \mu \mathrm{rad}$ rms.

A schematic of the LTP is shown in Figure 1. When the straightness reference was conceived, it was assumed that any slope errors coming from angle changes generated by the carriage would be removed from the SUT measurement. This included slope errors from laser pointing instability. Data from many measurements supported this assumption. However, after the practice of LTP measurements had improved significantly, small deviations in slope were noticed in both the SUT and REF slope functions. If these slope deviations were truly part of the SUT, then they would be seen in only the SUT measurement and not the REF measurement. If they originated from the carriage, then they would be seen in the REF measurement as well as in the SUT measurement, and could be subtracted from the SUT slope function.

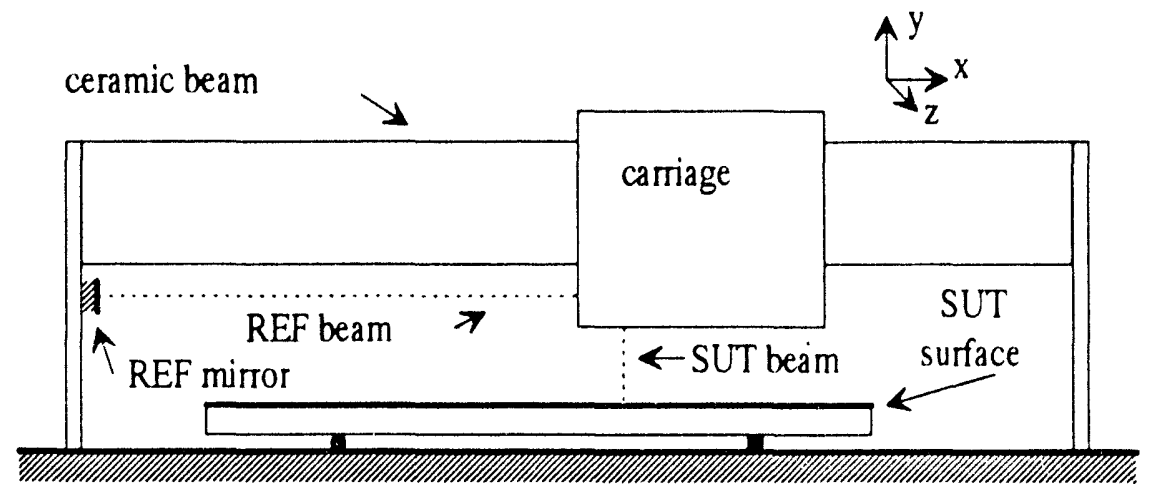

Figure 1. LTP schematic.

These small deviations were seen in both the REF and SUT measurements. However, when the REF function was subtracted from the SUT function so that carriage pitching errors would be removed, then the magnitude of these small deviations was increased in the resulting slope function. Apparently, there was a source of slope error manifest in the reference with magnitude or sign different 
than the carriage pitching errors. On "bad days" they would be very noticeable with magnitudes of about $1 \mu \mathrm{rad}$ and periods of around 2 to $20 \mathrm{~mm}$. On "good days" these deviations would be barely noticeable, and the functions would be very smooth.

\section{Angle analysis}

Figure 2 shows the geometrical layout of the main optical components of the LTP. The components which are part of the carriage are shown in the broken line. Light from the source $S$ (laser) propagates downward toward the beamsplitter $\mathrm{BS}$, where the light takes two paths. In one path, the light continues downward toward the SUT, reflects up toward BS, then reflects from BS into the lens system $\mathrm{L}$, which focuses the light onto the photodetector PD. In the other path, light is reflected toward REF, is reflected back through BS and into L and is focused onto PD.

Each of the SUT and REF beams produces a pattern that is imaged onto PD. The patterns are analyzed ${ }^{5}$ for each position $x$ along the SUT, and a resulting slope function of $x$ results. There is a slope function from the SUT and a slope function from the REF.

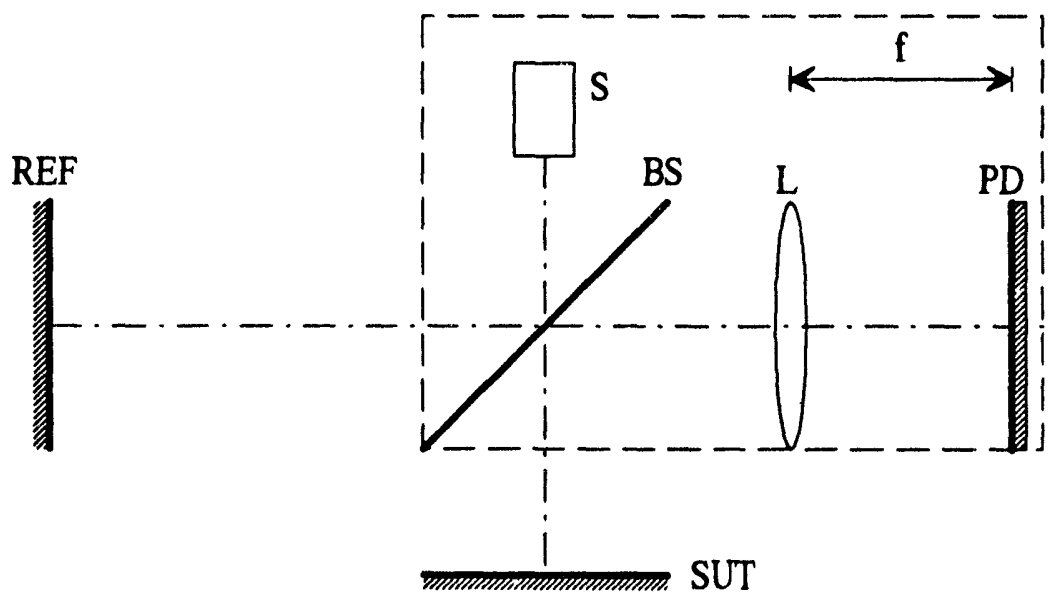

Figure 2. Nominal position of carriage components with respect to REF and SUT mirrors. 
Suppose the carriage is rotated clockwise by $\theta$. The relative angular change with respect to the REF and SUT mirrors is shown in Figure 3. Since the REF and SUT mirrors are each rotated counterclockwise by $\theta$ with respect to the carriage, then the light beams are reflected with a change of $2 \theta$. The REF beam angle is changed $+2 \theta$, while the SUT beam angle is changed $-2 \theta$ (clockwise) with respect to the main optic axis through $\mathrm{L}$ and $\mathrm{PD}$. Thus a pitch of the carriage causes twice the angular error in the SUT measurement and twice the angular error in the REF measurement; but the sign of error is reversed in the REF with respect to the SUT measurement.

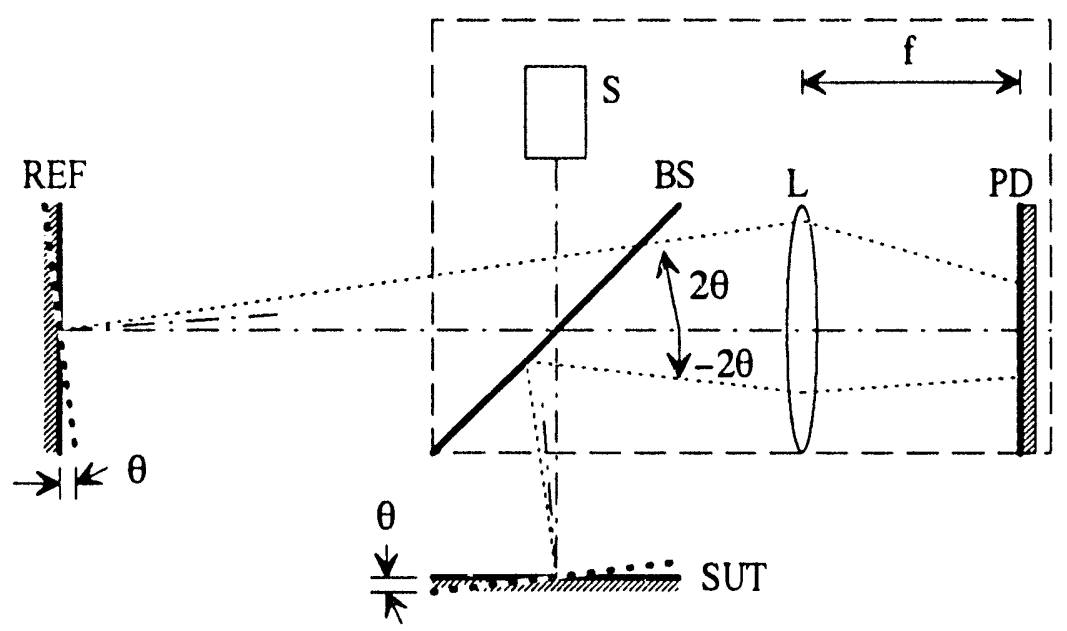

Figure 3. Carriage pitch of $\theta$ with respect to the REF and SUT mirrors.

Now the angle relationship between the SUT and REF measurements will be analyzed for a change in laser pointing. Figure 4 shows a trace of rays through the system as light from $S$ makes an angular change $\phi$. As seen from this figure, light reflects from BS toward the REF with an upward angular change $-\phi$. This angular change is reversed at the REF mirror, and thus produces an angular change $+\phi$ with respect to the main optic axis. At the same time, the light is reflected from the SUT with angular change $-\phi$ and then reflects from BS to produce an angular change $+\phi$ with respect to the main optic axis. Thus, a change in laser pointing causes the same angular change in the SUT measurement as in the REF measurement. 


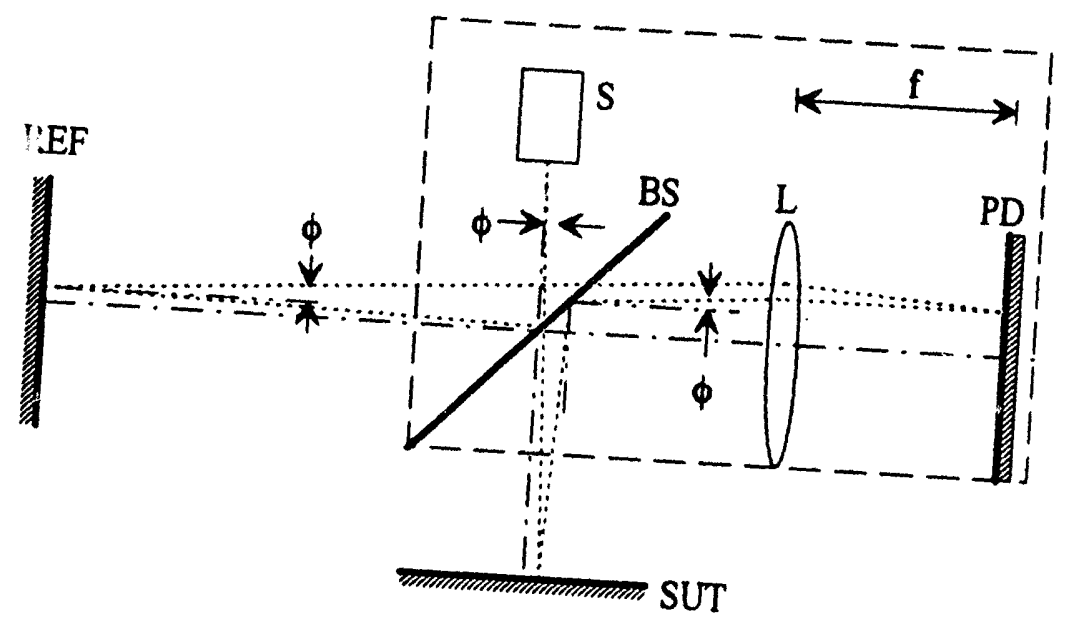

Figure 4. Analysis of laser pointing change $\phi$.

\section{Fixing the problem: a third slope function}

A straightforward way to fix the problem is to make use of the existing direction. If a beamsplitter BSe provide a third signal which indicates laser pointing REF beam path and tilted at a (fixed with respect to the carriage) is placed in the be imaged at one end of PD. As before, the in Figure 5, then the third pattern will The SUT pattern then is allowed to reside REF pattern is at the other end of PD. reference patterns. The third slope function from on the array between the two variations only as a result of angular beam changes this third pattern will then have

$x$ is the coordinate

slope function of the SUT for the carriage movement (see Figure 1). Let the true and let the carriage pitch error component ber pointing error component be $p(x)$, pattern from BSe is placed at the left end of PD. Now suppose that the third calculated from that pattern is $L(x)$. Also, the patten, and that the slope function two other patterns; let the slope function calculated from the SUT is between the Finally, suppose that the pattern from the REF is at thom that pattern be $M(x)$. resulting slope function is $R(x)$. The problem now the right end of $P D$, and its and sign) of $s(x), c(x)$, and $p(x)$ are in each of the is to find how much (magnitude and $R(x)$. 


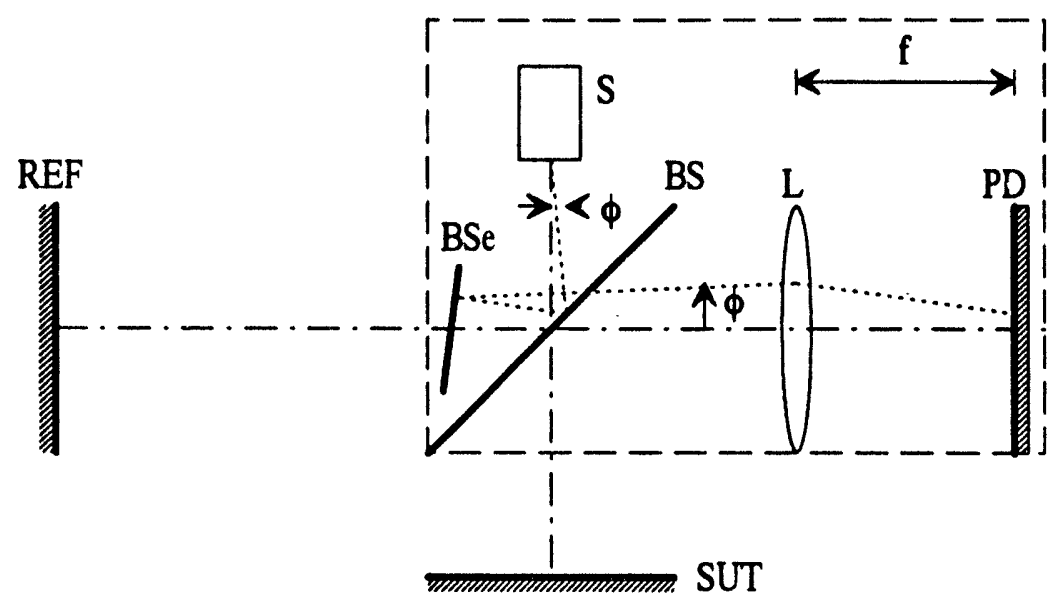

Figure 5. Addition of extra beamsplitter BSe.

It is seen from Figure 4 that the pattern $L(x)$ will consist entirely of $p(x)$. In keeping with the convention that counterclockwise angles are positive, a change of $\phi$ at $S$ produces a change $p(x)$ at PD. Thus

$$
L(x)=p(x) .
$$

Analysis of a change in $p(x)$ in the beam from $\mathrm{S}$ shows that upon reflection from the REF mirror, $R(x)$ will contain component $p(x)$. Furthermore, analysis of the effect of $c(x)$ shows that $R(x)$ also contains $+2 c(x)$. Thus

$$
R(x)=2 c(x)+p(x) .
$$

Likewise, similar analysis for the effect of $p(x)$ gives a component $p(x)$ in $M(x)$; the carriage pitching gives a component $-2 c(x)$ to $M(x)$ as well. The effect of actual slope change in the SUT causes the additional component in $M(x)$ of $-2 s(x)$. Thus

$$
M(x)=-2 s(x)-2 c(x)+p(x) .
$$

Equations (1), (2), and (3) are a set of three unknowns in three equations. Systematically solving them for $s(x)$ gives

$$
s(x)=[M(x)+R(x)-2 L(x)] /(-2) .
$$


Equation (4) gives the true slope function of the SUT in terms of the three measured slope functions. The factor -2 in the denominator reminds us that the optical beam angular change is twice the surface angular change, and that reflection of the SUT beam from BS causes an angular sign change.

\section{Sample measurement of a flat mirror}

Before the extra beamsplitter BSe was employed, a typical data processing session for a mirror would consist of subtracting the REF signal from the SUT signal, i.e. adding $R(x)$ to $M(x)$ and dividing by -2 to obtain $s(x)$. Figure 6 shows the result of measuring a flat (assured to be better than $\lambda / 50$ ) mirror, and processing the data in this fashion. Mid-period variations are seen to be fairly pronounced, and there is significant curvature.

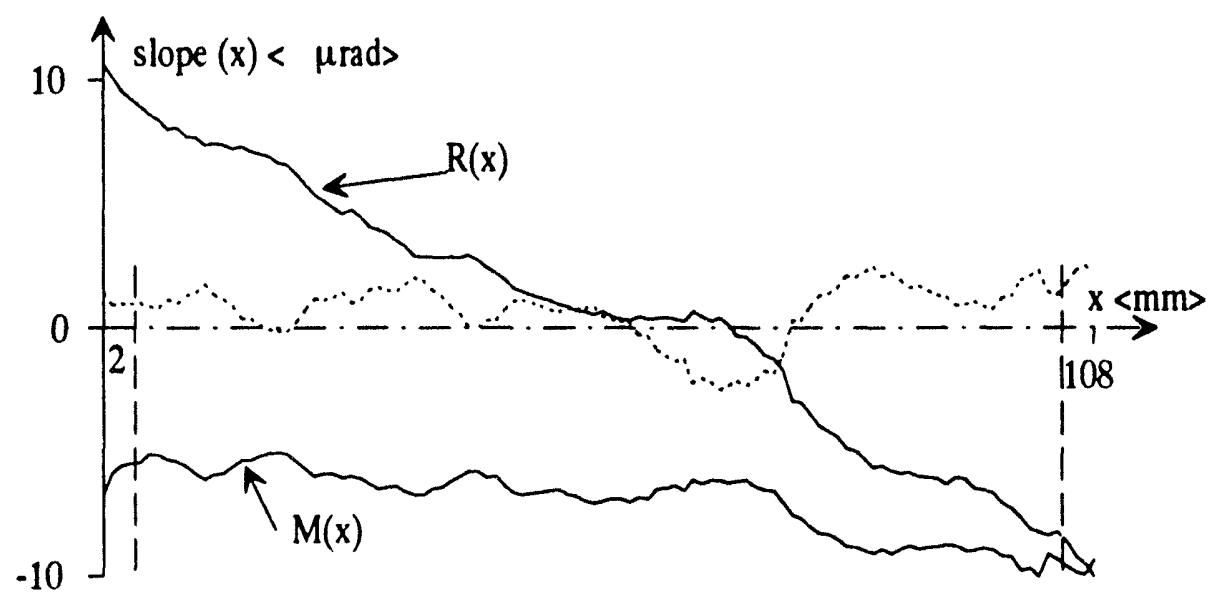

Figure 6. Measurement of a flat mirror without use of the extra beamsplitter. $s(x)$ is shown as the dotted line. variation $=1.32 \mu \mathrm{rad} \mathrm{rms} ; \mathrm{R}=4892 \mathrm{~m}$. Clear aperture ( $5 \mathrm{~mm}$ from edge) for calculations is between the vertical broken lines.

These measurements were then processed using the extra beamsplitter and the formula (4). The results are shown in Figure 7. Notice that now the edge rolloff from polishing is visible at the ends of the measurement scan. The midperiod variation is significantly reduced, and the resulting variation is below 0.5 $\mu \mathrm{rad}$. Measured curvature is also much less. 


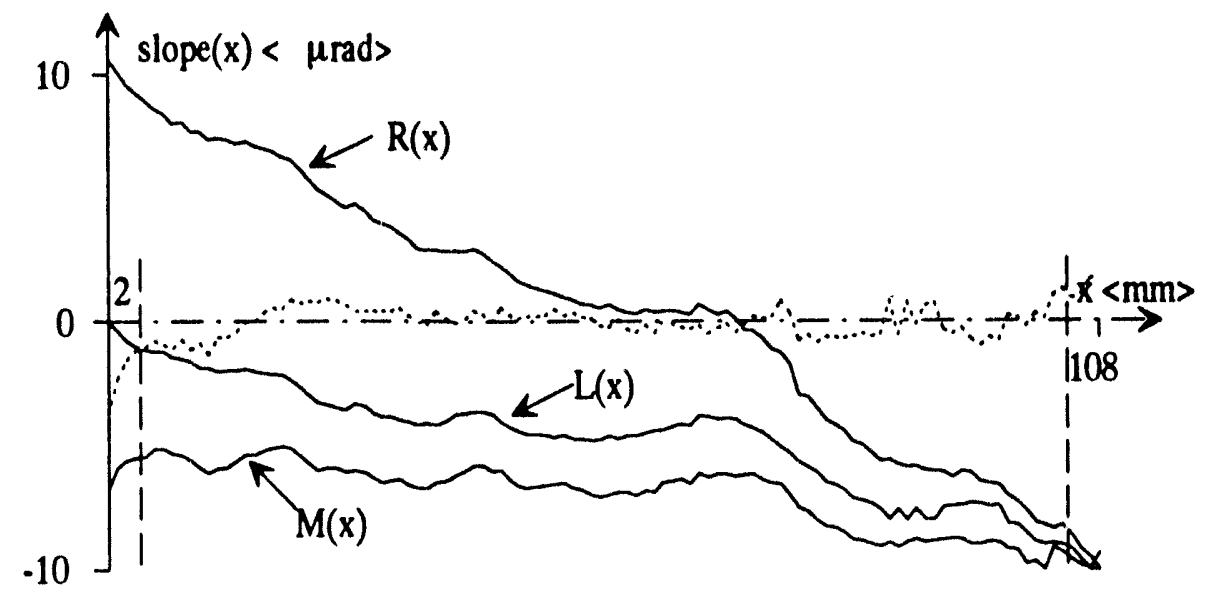

Figure 7. Measurement of a flat mirror with use of the extra beamsplitter. $s(x)$ is shown as the dotted line. variation $=0.454 \mu \mathrm{rad} \mathrm{rms} ; \mathrm{R}=-14059 \mathrm{~m}$. Clear aperture ( $5 \mathrm{~mm}$ from edge) for calculations is between the vertical broken lines.

Curvature of the surface is manifested as degree of tilt in the slope plot. Thus in Figure 6 much of the curvature is seen to be from $R(x)$. Using the previous data processing procedure, it could only be assumed that curvature in $R(x)$ is due entirely to $c(x)$ (sag in the ceramic beam, for example). After data processing, $s(x)$ would contain the extra curvature seen in $R(x)$. Figure 7 shows that the gradual laser pointing drift $p(x)$ (manifest in $L(x)$ ) is largely responsible for the extra curvature in $R(x)$. Applying the processing formula (4) significantly reduces the curvature that would otherwise be in $s(x)$.

The measurements presented here are neither the best nor the worst of all measurements made on this mirror. The author believes them to be typical. However, careful preparation was made for this measurement. Metrology of this scale requires acute attention to mounting methods and temperature control on the order of 0.1 degrees Centigrade over the time of the measurement scan.

\section{Conclusion}

The problem of identifying and analyzing a source of error (laser pointing change) in slope measurement using an LTP has been discussed. One method of 
correcting for the laser pointing change has been described, and the results of this correction are encouraging. It is desirable to reduce the amount of pointing change in any case, and this might be done by transferring the light from a laser via a single mode fiber to the position indicated by $S$ in the above figures. Evaluation of this idea is pending.

\section{Acknowledgments}

Peter Takacs (Brookhaven National Laboratory) is to be thanked for his contribution of the laser-to-fiber idea. Also, discussion of temperature control in metrology with Chris Evans (NIST) is much appreciated. This work was supported by the Director, Office of Energy Research, Office of Basic Energy Sciences, Materials Sciences Division of the U. S. Department of Energy, Under contract No. DE-AC03-76SF00098.

\section{References}

1. Takacs, P. Z., and Qian, S, United States Patent 4884697, 1989.

2. Takacs, P. Z., Feng, S. K., Church, E. L., Qian, S., and Liu, W., "Long trace profile measurements on cylindrical Aspheres," Proc.SPIE, vol. 966, (1988) 354.

3. Irick, S. C., McKinney, W. R., Lunt, D. L. J., and Takacs, P. Z., "Using a straightness reference in obtaining more accurate surface profiles from a long trace profiler," Review of Scientific Instruments, vol. 63, No.1 (Part IIB), 1436-1438, (January, 1992).

4. Irick, S. C., "Advancements in one-dimensional profiling with a long trace profiler", Proc. SPIE, vol. 1720, (1992), 162.

5. Irick, S. C., "Determining surface profile from sequential interference patterns from a long trace profiler," Review of Scientific Instruments, vol. 63, No.1 (Part IIB), 1432-1435, (January, 1992). 

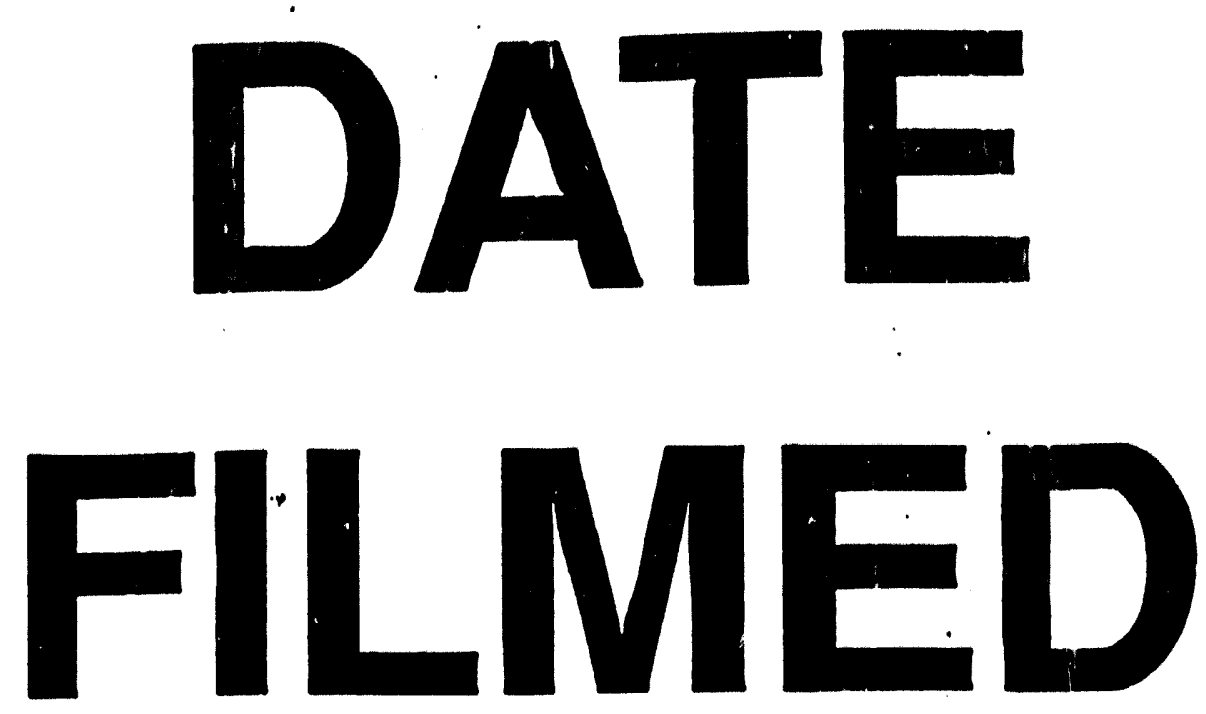

$1 / 21 / 94$
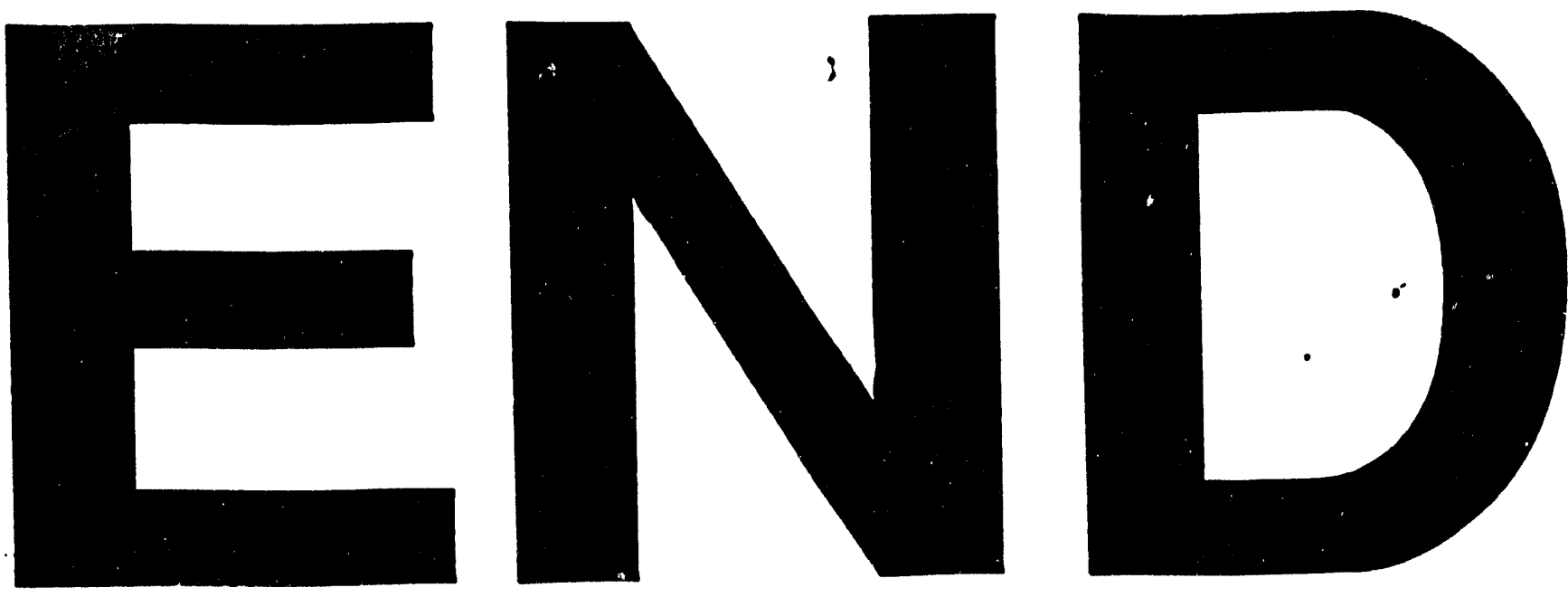


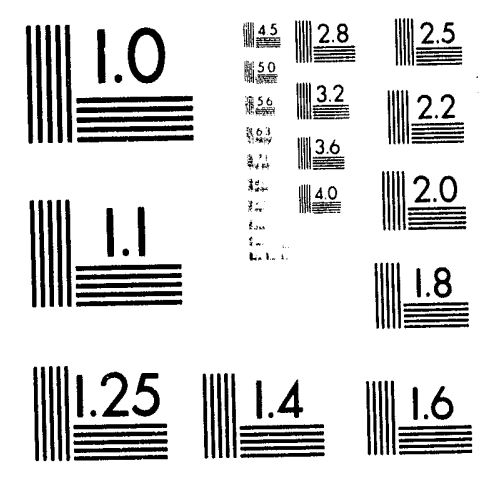



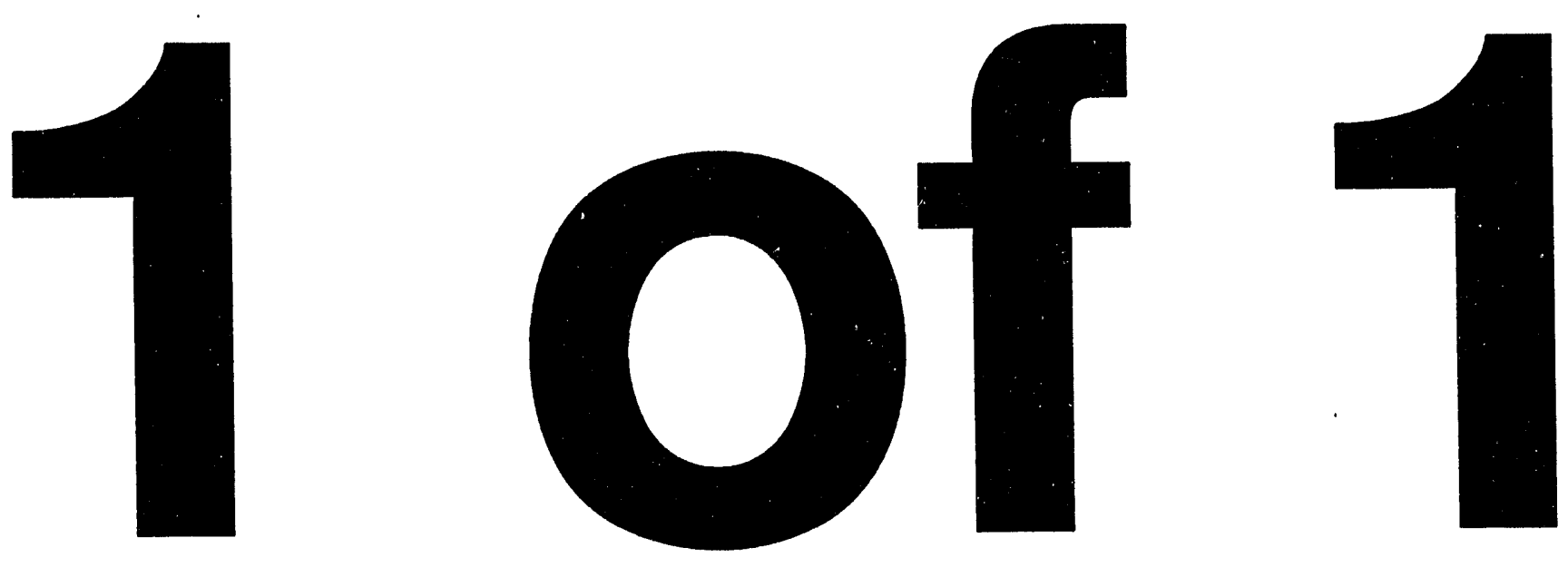


\title{
IMPROVED MEASUREMENT ACCURACY IN A LONG TRACE PROFILER: COMPENSATION FOR LASER POINTING INSTABILITY
}

\author{
S. Irick
}

\author{
Advanced Light Source \\ Accelerator and Fusion Research Division \\ Lawrence Berkeley Laboratory \\ University of California \\ Berkeley,CA 94720
}

August 2, 1993

Paper Presented at the 8th National Conference on Synchrotron Radiation Instrumentation, Gaithersburg, Maryland, USA, 8/23/93-8/26/93. 


\title{
Improved Measurement Accuracy in a Long Trace Profiler: Compensation for Laser Pointing Instability
}

\author{
Steven C. Irick \\ Lawrence Berkeley Laboratory \\ University of California \\ 1 Cyclotron Road \\ Berkeley, Ca 94720
}

\begin{abstract}
Laser pointing instability adds to the error of slope measurements taken with the Long Trace Profiler ${ }^{1}$ (LTP). As with carriage pitch error, this laser pointing error must be accounted for and subtracted from the surface under test (SUT) slope measurement. In the past, a separate reference beam (REF) allowed characterization of the component of slope error from carriage pitch. However, the component of slope error from laser pointing manifests itself differently in the SUT measured slope.

An analysis of angle error propagation is given, and the effect of these errors on measured slope is determined. Then a method is proposed for identifying these errors and subtracting them from the measured SUT slope function. Separate measurements of carriage pitch and laser pointing instability isolate these effects, so that the effectiveness of the error identification algorithm may be demonstrated.
\end{abstract}

\section{Introduction}

Ever since the LTP was first used for measuring long mirrors of arbitrary figure, there has been an on-going effort to increase the accuracy and reduce inherent noise of this instrument. The first LTP built by Takacs et al. ${ }^{2}$ provided a means of measuring this class of optical components that no commercially available instrument could provide. At that time it was impressive to have slope features in the sub-milliradian (mrad) regime revealed. 
More recently, high-brightness synchrotrons have pushed requirements of optical components in beamlines to preserve the brightness. Third-generation synchrotron beamlines have produced designs which specify slope errors of less than one microradian ( $\mu \mathrm{rad})$ rms. In order to achieve this goal, a straightness reference $^{3}$ was employed, which compensated for errors in carriage movement. An improved air bearing stage, environment control, and improved mounting methods ${ }^{4}$ also reduced the noise level, so that slope errors of $160 \mathrm{~mm}$ long mirrors could be measured with accuracies better than $1 \mu \mathrm{rad}$ rms.

A schematic of the LTP is shown in Figure 1. When the straightness reference was conceived, it was assumed that any slope errors coming from angle changes generated by the carriage would be removed from the SUT measurement. This included slope errors from laser pointing instability. Data from many measurements supported this assumption. However, after the practice of LTP measurements had improved significantly, small deviations in slope were noticed in both the SUT and REF slope functions. If these slope deviations were truly part of the SUT, then they would be seen in only the SUT measurement and not the REF measurement. If they originated from the carriage, then they would be seen in the REF measurement as well as in the SUT measurement, and could be subtracted from the SUT slope function.

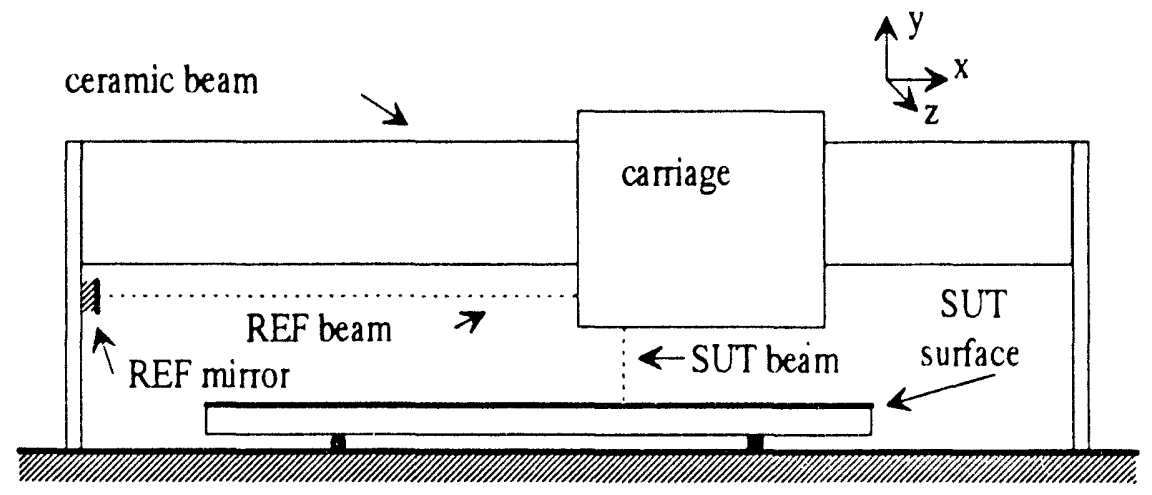

Figure 1. LTP schematic.

These small deviations were seen in both the REF and SUT measurements. However, when the REF function was subtracted from the SUT function so that carriage pitching errors would be removed, then the magnitude of these small deviations was increased in the resulting slope function. Apparently, there was a source of slope error manifest in the reference with magnitude or sign different 
than the carriage pitching errors. On "bad days" they would be very noticeable with magnitudes of about $1 \mu \mathrm{rad}$ and periods of around 2 to $20 \mathrm{~mm}$. On "good days" these deviations would be barely noticeable, and the functions would be very smooth.

\section{Angle analysis}

Figure 2 shows the geometrical layout of the main optical components of the LTP. The components which are part of the carriage are shown in the broken line. Light from the source $S$ (laser) propagates downward toward the beamsplitter $\mathrm{BS}$, where the light takes two paths. In one path, the light continues downward toward the SUT, reflects up toward BS, then reflects from BS into the lens system $\mathrm{L}$, which focuses the light onto the photodetector PD. In the other path, light is reflected toward REF, is reflected back through BS and into L and is focused onto PD.

Each of the SUT and REF beams produces a pattern that is imaged onto PD. The patterns are analyzed ${ }^{5}$ for each position $x$ along the SUT, and a resulting slope function of $x$ results. There is a slope function from the SUT and a slope function from the REF.

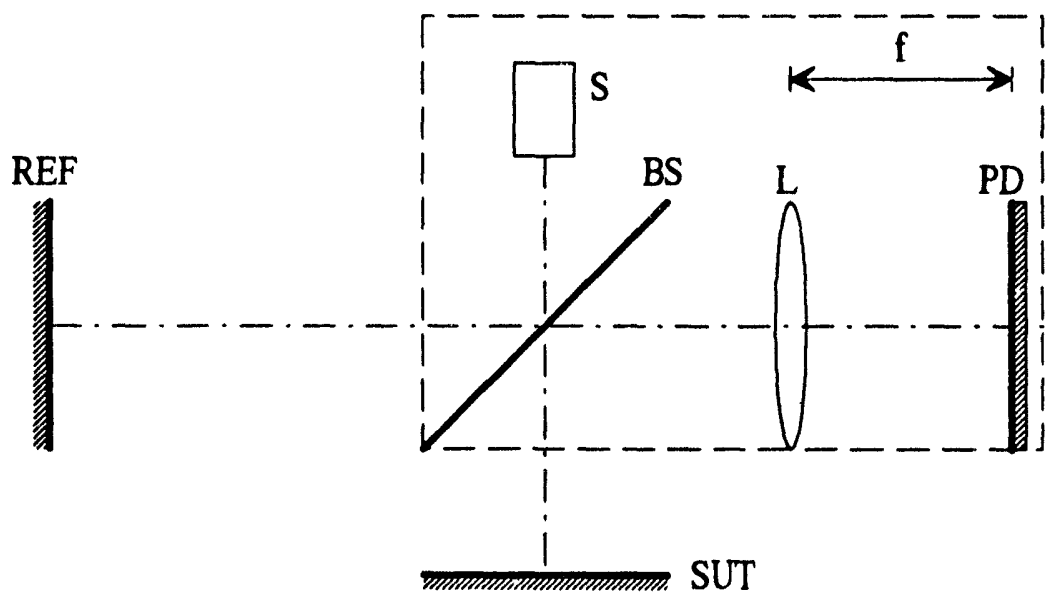

Figure 2. Nominal position of carriage components with respect to REF and SUT mirrors. 
Suppose the carriage is rotated clockwise by $\theta$. The relative angular change with respect to the REF and SUT mirrors is shown in Figure 3. Since the REF and SUT mirrors are each rotated counterclockwise by $\theta$ with respect to the carriage, then the light beams are reflected with a change of $2 \theta$. The REF beam angle is changed $+2 \theta$, while the SUT beam angle is changed $-2 \theta$ (clockwise) with respect to the main optic axis through $\mathrm{L}$ and $\mathrm{PD}$. Thus a pitch of the carriage causes twice the angular error in the SUT measurement and twice the angular error in the REF measurement; but the sign of error is reversed in the REF with respect to the SUT measurement.

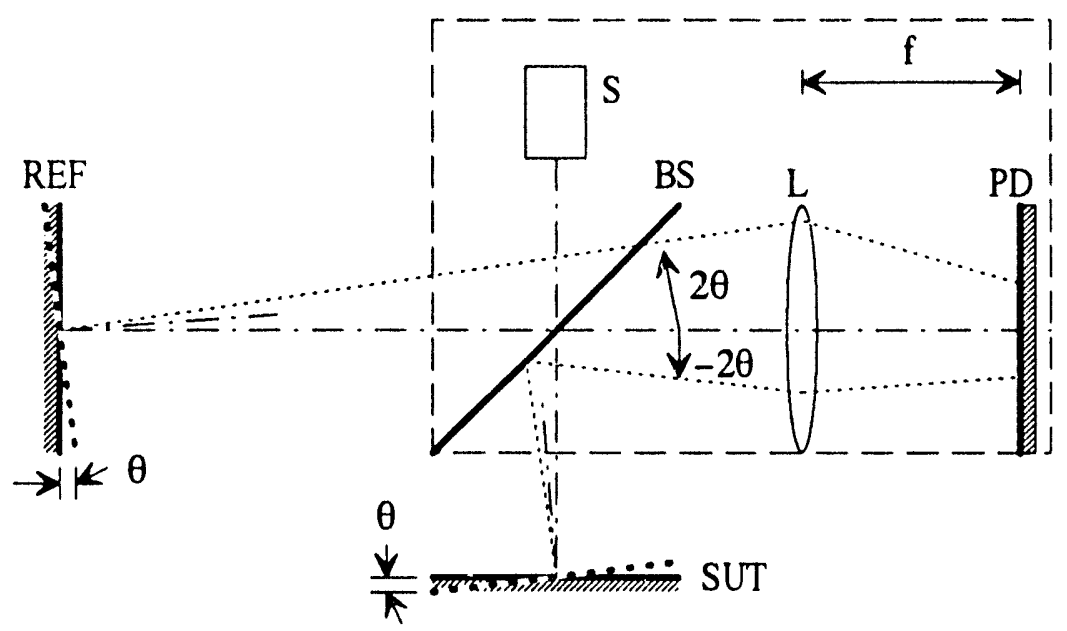

Figure 3. Carriage pitch of $\theta$ with respect to the REF and SUT mirrors.

Now the angle relationship between the SUT and REF measurements will be analyzed for a change in laser pointing. Figure 4 shows a trace of rays through the system as light from $S$ makes an angular change $\phi$. As seen from this figure, light reflects from BS toward the REF with an upward angular change $-\phi$. This angular change is reversed at the REF mirror, and thus produces an angular change $+\phi$ with respect to the main optic axis. At the same time, the light is reflected from the SUT with angular change $-\phi$ and then reflects from BS to produce an angular change $+\phi$ with respect to the main optic axis. Thus, a change in laser pointing causes the same angular change in the SUT measurement as in the REF measurement. 


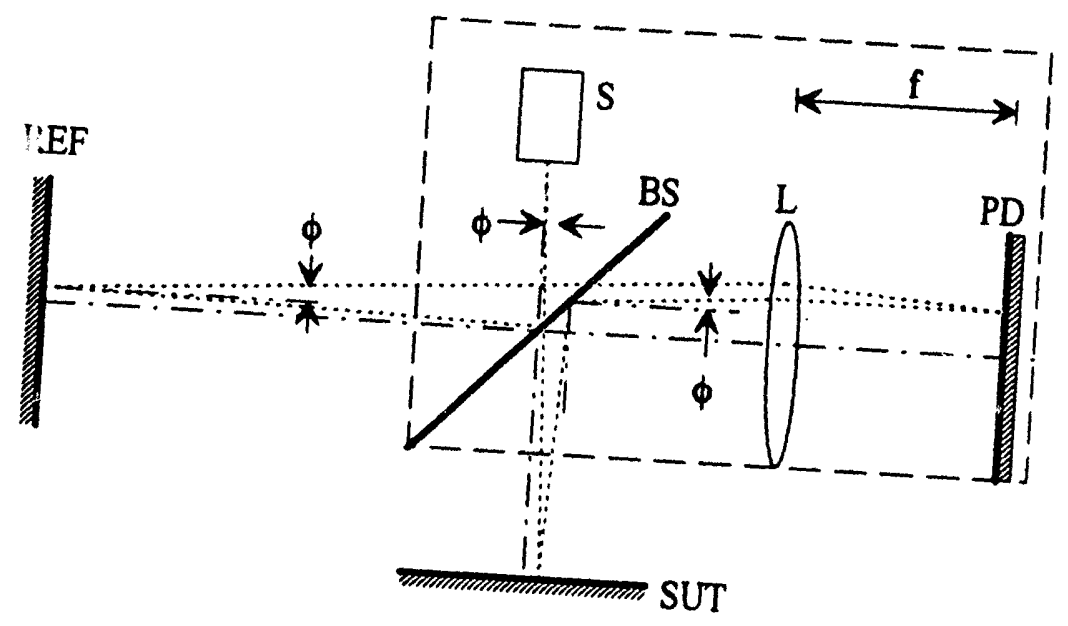

Figure 4. Analysis of laser pointing change $\phi$.

\section{Fixing the problem: a third slope function}

A straightforward way to fix the problem is to make use of the existing direction. If a beamsplitter BSe provide a third signal which indicates laser pointing REF beam path and tilted at a (fixed with respect to the carriage) is placed in the be imaged at one end of PD. As before, the in Figure 5, then the third pattern will The SUT pattern then is allowed to reside REF pattern is at the other end of PD. reference patterns. The third slope function from on the array between the two variations only as a result of angular beam changes this third pattern will then have

$x$ is the coordinate

slope function of the SUT for the carriage movement (see Figure 1). Let the true and let the carriage pitch error component ber pointing error component be $p(x)$, pattern from BSe is placed at the left end of PD. Now suppose that the third calculated from that pattern is $L(x)$. Also, the patten, and that the slope function two other patterns; let the slope function calculated from the SUT is between the Finally, suppose that the pattern from the REF is at thom that pattern be $M(x)$. resulting slope function is $R(x)$. The problem now the right end of $P D$, and its and sign) of $s(x), c(x)$, and $p(x)$ are in each of the is to find how much (magnitude and $R(x)$. 


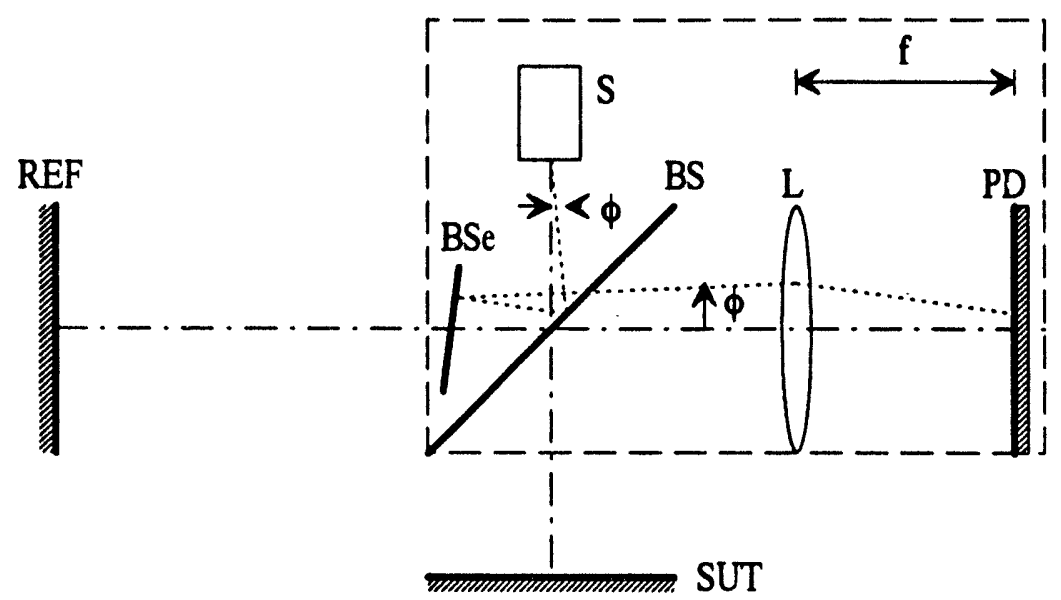

Figure 5. Addition of extra beamsplitter BSe.

It is seen from Figure 4 that the pattern $L(x)$ will consist entirely of $p(x)$. In keeping with the convention that counterclockwise angles are positive, a change of $\phi$ at $S$ produces a change $p(x)$ at PD. Thus

$$
L(x)=p(x) .
$$

Analysis of a change in $p(x)$ in the beam from $\mathrm{S}$ shows that upon reflection from the REF mirror, $R(x)$ will contain component $p(x)$. Furthermore, analysis of the effect of $c(x)$ shows that $R(x)$ also contains $+2 c(x)$. Thus

$$
R(x)=2 c(x)+p(x) .
$$

Likewise, similar analysis for the effect of $p(x)$ gives a component $p(x)$ in $M(x)$; the carriage pitching gives a component $-2 c(x)$ to $M(x)$ as well. The effect of actual slope change in the SUT causes the additional component in $M(x)$ of $-2 s(x)$. Thus

$$
M(x)=-2 s(x)-2 c(x)+p(x) .
$$

Equations (1), (2), and (3) are a set of three unknowns in three equations. Systematically solving them for $s(x)$ gives

$$
s(x)=[M(x)+R(x)-2 L(x)] /(-2) .
$$


Equation (4) gives the true slope function of the SUT in terms of the three measured slope functions. The factor -2 in the denominator reminds us that the optical beam angular change is twice the surface angular change, and that reflection of the SUT beam from BS causes an angular sign change.

\section{Sample measurement of a flat mirror}

Before the extra beamsplitter BSe was employed, a typical data processing session for a mirror would consist of subtracting the REF signal from the SUT signal, i.e. adding $R(x)$ to $M(x)$ and dividing by -2 to obtain $s(x)$. Figure 6 shows the result of measuring a flat (assured to be better than $\lambda / 50$ ) mirror, and processing the data in this fashion. Mid-period variations are seen to be fairly pronounced, and there is significant curvature.

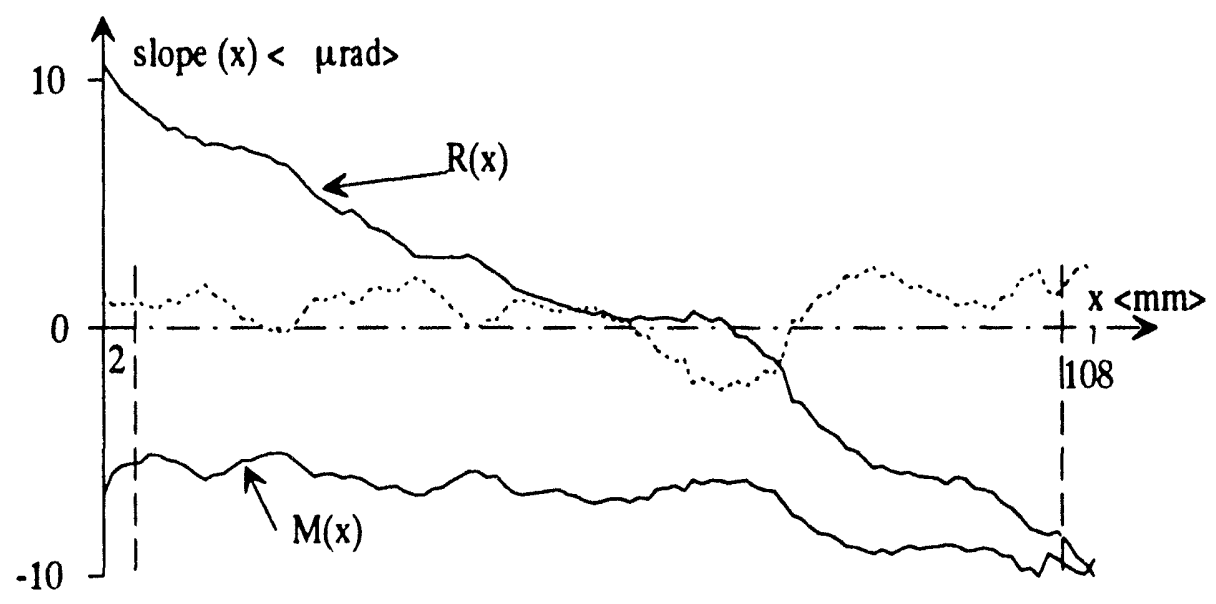

Figure 6. Measurement of a flat mirror without use of the extra beamsplitter. $s(x)$ is shown as the dotted line. variation $=1.32 \mu \mathrm{rad} \mathrm{rms} ; \mathrm{R}=4892 \mathrm{~m}$. Clear aperture ( $5 \mathrm{~mm}$ from edge) for calculations is between the vertical broken lines.

These measurements were then processed using the extra beamsplitter and the formula (4). The results are shown in Figure 7. Notice that now the edge rolloff from polishing is visible at the ends of the measurement scan. The midperiod variation is significantly reduced, and the resulting variation is below 0.5 $\mu \mathrm{rad}$. Measured curvature is also much less. 


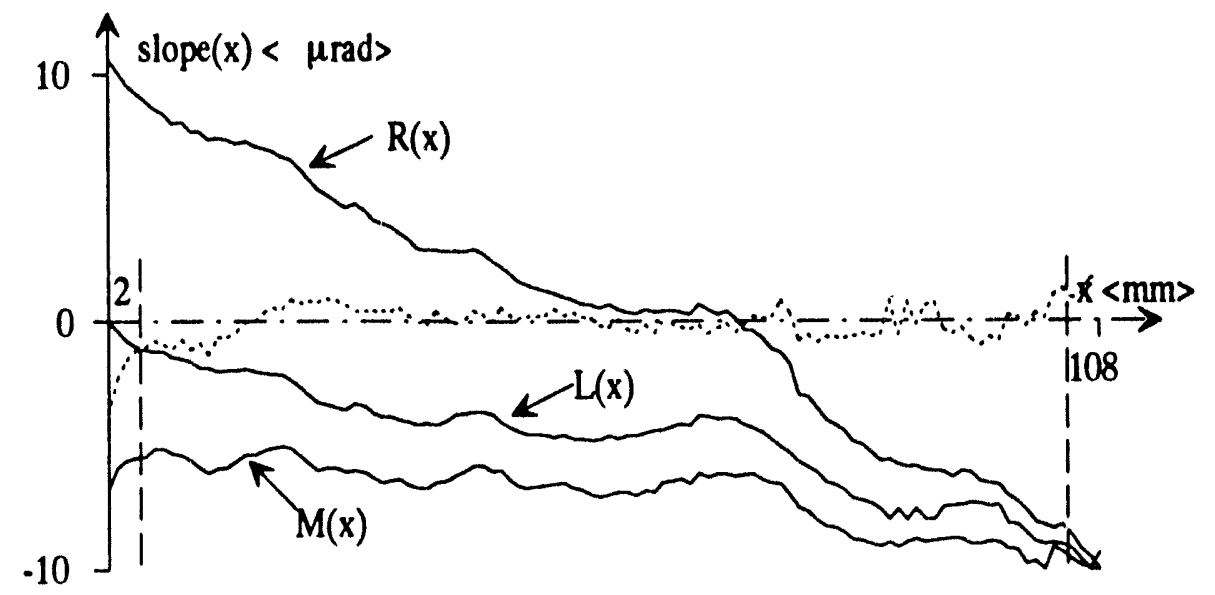

Figure 7. Measurement of a flat mirror with use of the extra beamsplitter. $s(x)$ is shown as the dotted line. variation $=0.454 \mu \mathrm{rad} \mathrm{rms} ; \mathrm{R}=-14059 \mathrm{~m}$. Clear aperture ( $5 \mathrm{~mm}$ from edge) for calculations is between the vertical broken lines.

Curvature of the surface is manifested as degree of tilt in the slope plot. Thus in Figure 6 much of the curvature is seen to be from $R(x)$. Using the previous data processing procedure, it could only be assumed that curvature in $R(x)$ is due entirely to $c(x)$ (sag in the ceramic beam, for example). After data processing, $s(x)$ would contain the extra curvature seen in $R(x)$. Figure 7 shows that the gradual laser pointing drift $p(x)$ (manifest in $L(x)$ ) is largely responsible for the extra curvature in $R(x)$. Applying the processing formula (4) significantly reduces the curvature that would otherwise be in $s(x)$.

The measurements presented here are neither the best nor the worst of all measurements made on this mirror. The author believes them to be typical. However, careful preparation was made for this measurement. Metrology of this scale requires acute attention to mounting methods and temperature control on the order of 0.1 degrees Centigrade over the time of the measurement scan.

\section{Conclusion}

The problem of identifying and analyzing a source of error (laser pointing change) in slope measurement using an LTP has been discussed. One method of 
correcting for the laser pointing change has been described, and the results of this correction are encouraging. It is desirable to reduce the amount of pointing change in any case, and this might be done by transferring the light from a laser via a single mode fiber to the position indicated by $S$ in the above figures. Evaluation of this idea is pending.

\section{Acknowledgments}

Peter Takacs (Brookhaven National Laboratory) is to be thanked for his contribution of the laser-to-fiber idea. Also, discussion of temperature control in metrology with Chris Evans (NIST) is much appreciated. This work was supported by the Director, Office of Energy Research, Office of Basic Energy Sciences, Materials Sciences Division of the U. S. Department of Energy, Under contract No. DE-AC03-76SF00098.

\section{References}

1. Takacs, P. Z., and Qian, S, United States Patent 4884697, 1989.

2. Takacs, P. Z., Feng, S. K., Church, E. L., Qian, S., and Liu, W., "Long trace profile measurements on cylindrical Aspheres," Proc.SPIE, vol. 966, (1988) 354.

3. Irick, S. C., McKinney, W. R., Lunt, D. L. J., and Takacs, P. Z., "Using a straightness reference in obtaining more accurate surface profiles from a long trace profiler," Review of Scientific Instruments, vol. 63, No.1 (Part IIB), 1436-1438, (January, 1992).

4. Irick, S. C., "Advancements in one-dimensional profiling with a long trace profiler", Proc. SPIE, vol. 1720, (1992), 162.

5. Irick, S. C., "Determining surface profile from sequential interference patterns from a long trace profiler," Review of Scientific Instruments, vol. 63, No.1 (Part IIB), 1432-1435, (January, 1992). 

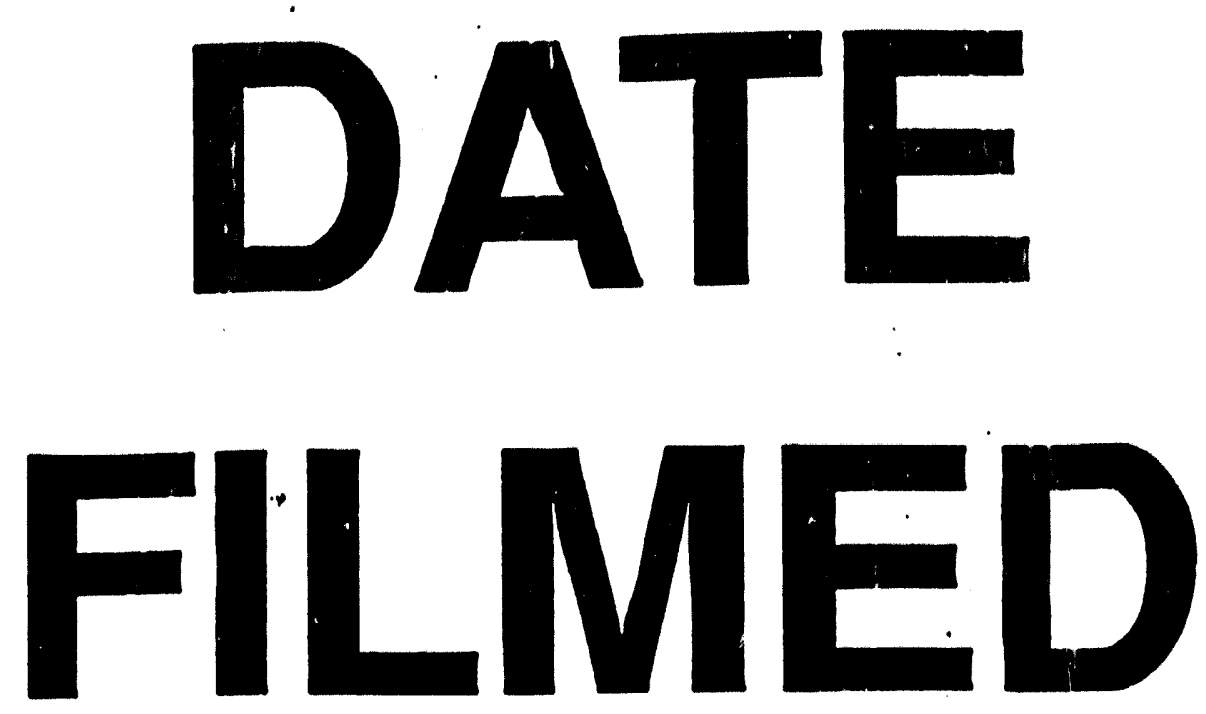

$1 / 21 / 94$
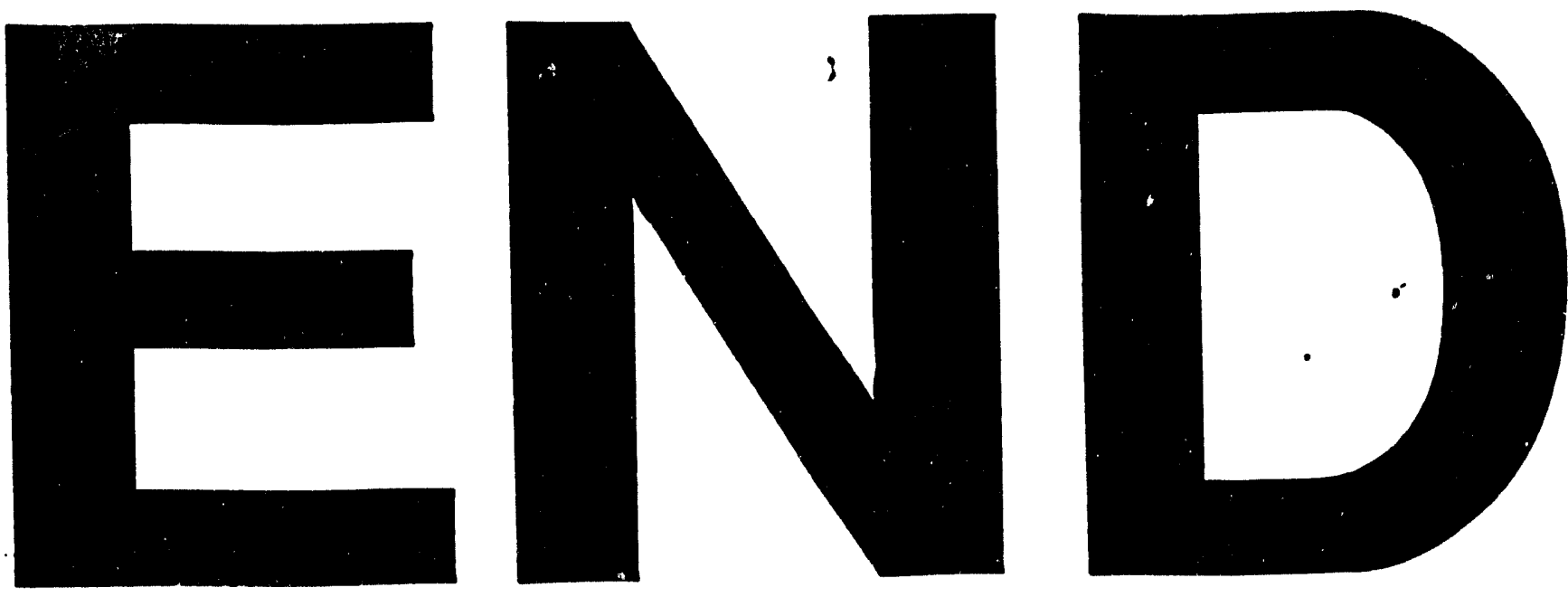